\title{
An unusual cause of dyspareunia: secondary cervical perforation by post placental intrauterine contraceptive device
}

\author{
Monica Chauhan*, Gita Guin
}

Department of Obstetrics \& Gynecology, NSCB Government Medical College \& Hospital, Jabalpur, M.P., India

Received: 19 March 2015

Accepted: 19 April 2015

\author{
*Correspondence: \\ Dr. Monica Chauhan, \\ E-mail: drmonicachauhan@gmail.com
}

Copyright: () the author(s), publisher and licensee Medip Academy. This is an open-access article distributed under the terms of the Creative Commons Attribution Non-Commercial License, which permits unrestricted non-commercial use, distribution, and reproduction in any medium, provided the original work is properly cited.

\begin{abstract}
Postpartum contraception using intrauterine device Copper T $380 \mathrm{~A}$ is considered a valuable option to tackle high rate of unintended pregnancy. This case is presented to highlight the importance of follow up and a vigilant examination on patient reporting with any complaint on follow up, even in the presence of a normally placed thread which is crucial to identify problem and manage complication before this reliable, cost effective and long term postpartum contraceptive solution fails to see its rise.
\end{abstract}

Keywords: Postpartum intrauterine contraceptive device, Cervical perforation, Dyspareunia

\section{INTRODUCTION}

In India, $65 \%$ of women in the first year postpartum have an unmet need for family planning and $61 \%$ of births occur at shorter interval of recommended birth to birth interval. ${ }^{1}$ To deal with the problem Government is emphasizing on postpartum long term contraception. With the standardization of postpartum contraceptive insertion technique it has shown good efficacy with minimal complications. Intrauterine devices are most emphasized one time long term temporary means of postpartum contraception in the developing countries.

The frequently encountered symptoms are menstrual irregularity, dysmenorrhea, and spontaneous expulsion, only rarely are postpartum intrauterine devices misplaced because of improvements in insertion technique and with clear cut guidelines. ${ }^{2}$ Misplaced intrauterine device causing distressing dyspareunia in the presence of a normally placed thread is rarely reported. We are reporting a case of a secondary cervical perforation due to copper T 380 A as cause of dyspareunia. This may be partly attributed to misconception related to intrauterine device usage and myths surrounding high chance of perforation.

\section{CASE REPORT}

24 years old primiparous woman had last child birth 6 months back came with complaints of dyspareunia and pain on squatting for last 3 months. She had an uneventful vaginal delivery in our tertiary care hospital followed by immediate (within 10 minutes) post placental insertion of $\mathrm{Cu} \mathrm{T} 380 \mathrm{~A}$ after consenting and with advise to return to the clinic for routine postpartum care at $6^{\text {th }}$ week. She had a routine follow up visit at $6^{\text {th }}$ week with no symptom and normal findings.

Since resuming coitus 3 months back patient started experiencing distressing dyspareunia and pain on squatting. She reported to our Gynecology OPD for the same complaints and was examined by a resident; since the patient had no menstrual complaints and cu thread was seen coming through the cervical os she was advised antibiotics and non-steroidal anti-inflammatory drugs. Unrelieved of her symptoms she again reported to the 
OPD after 15 days. On careful speculum examination IUCD thread was seen coming through cervical os, the knob of the copper covered vertical limb was seen peeping through the posterior aspect of the cervix (Figure 1). Patient was explained about the present condition and counseled for removal. After obtaining consent, gentle traction on the knob easily dislodged the IUD which was pulled out holding the vertical limb (Figure 2). There was no bleeding and patient was sent home the same day. Patient was relieved at one week follow up but refused immediate alternative contraception, despite counseling.

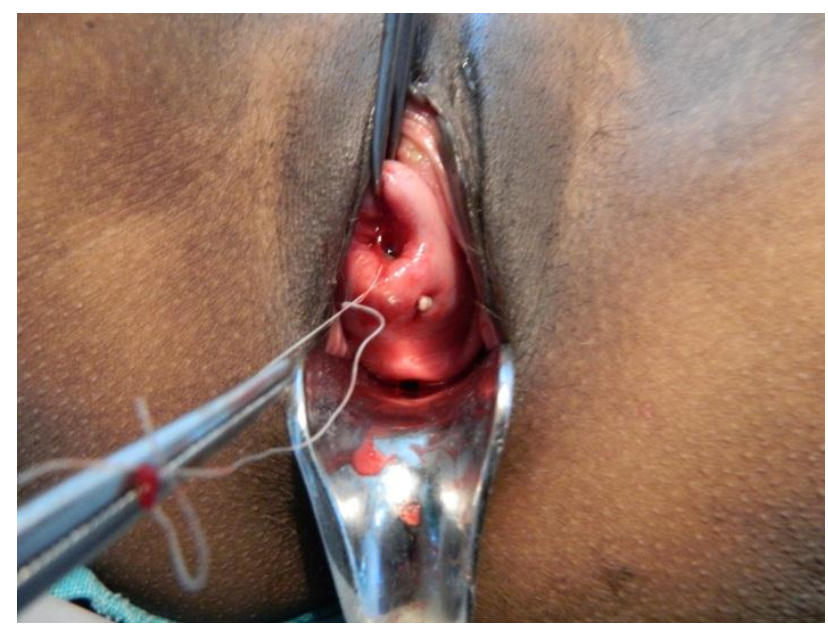

Figure 1: Secondary cervical perforation by post placental intrauterine device.

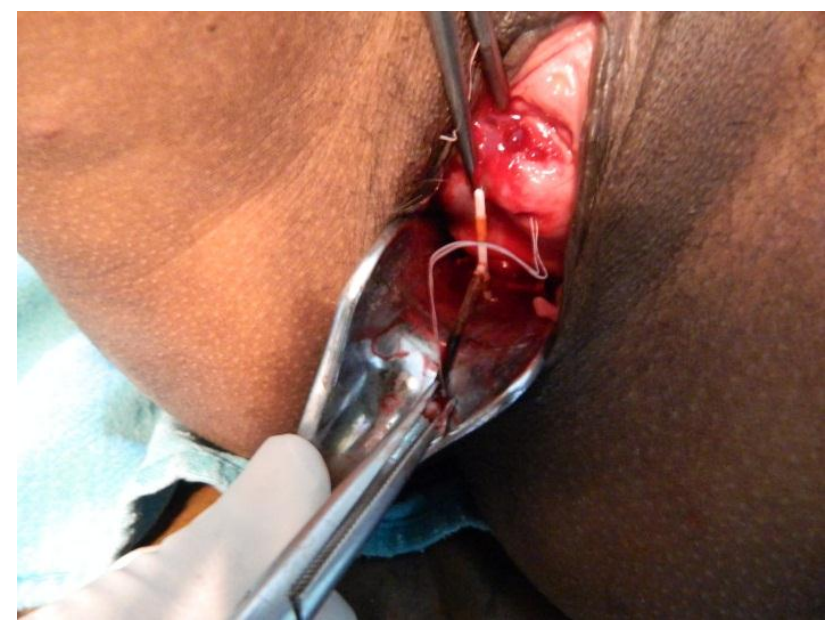

Figure 2: Removal of Copper $\mathrm{T}$ by holding the knob of vertical limb.

\section{DISCUSSION}

Dyspareunia in the early period following child birth is commonly attributed to psychological (anxiety), pelvic inflammatory disease, uterine retroversion, chronic constipation with fissure in ano and perineal pain. The problems associated with intrauterine contraceptive device is menstrual irregularity the absence of which usually assures the clinician of a well-accepted intrauterine device, while displacements is less reported with postpartum insertion and it is usually correlated with a missing thread. The finding of a thread protruding through the cervical os is an assurance to clinician of IUD being in place. Secondary Cervical perforation of intrauterine device has been reported in the literature but only a fraction had symptoms. Since the thread was seen emerging through the os in almost all cases therefore even when the thread is in place, it is mandatory to do a careful inspection of fornices to exclude a perforated device. This is important in view of increased chance of contraceptive failure leading to an unwanted pregnancy to the subject and litigation for the service provider. Cervical perforation is a rare but well described hazard. ${ }^{3}$ Supervised training and adherence to manual guidelines are prerequisites for safe practice but what is important is need based follow up and prompt management of complication.

Postpartum intrauterine device has fewer incidences of complications but comparatively high incidence of spontaneous expulsion. ${ }^{4}$ Women opting for this method of contraception should be counseled for possibility of spontaneous expulsion, risk of perforation, menstrual irregularity and importance of follow up.

\section{CONCLUSION}

Women attending for a postpartum Copper $\mathrm{T}$ checkup with symptoms even in a presence of a normally placed thread should be critically evaluated and with a low threshold for initiating further investigation. The success of popularizing postpartum insertion of intrauterine Copper $\mathrm{T}$ following institutional deliveries hinges on strict adherence to the criteria laid down for patient selection and insertion and rigorous follow up to diagnose and manage any complication so as to not harm the popularity of this very effective method of contraception. ${ }^{5}$

\section{Funding: No funding sources Conflict of interest: None declared Ethical approval: Not required}

\section{REFERENCES}

1. Somesh Kumar, Reena Sethi, Sudharsanam Balasubramaniam, Elaine Charurat, Kamlesh Lalchandani, Richard Semba, et al. Women's experience with postpartum intrauterine contraceptive device use in India. Reprod Health. 2014; $11: 32$.

2. Government of India. Postpartum IUCD. In: GOI, eds. Reference Manual. India: Family Planning Division, Ministry of Health and Family Welfare; November 2010.

3. Ovadia J, Reichman J, Goldman JA. Secondary cervical perforation by the Copper-T intrauterine device. Eur J Obstet Gynecol Reprod Biol. 1979 Dec;9(6):403-4. 
4. Nathalie Kappa, Kathryn M. Curtis. Intrauterine device insertion during the postpartum period: a systematic review. Contraception. 2009;80:327-36.

5. Sahaja Kittur, Y. M. Kabadi. Enhancing contraceptive usage by post-placental intrauterine contraceptive devices (PPIUCD) insertion with evaluation of safety, efficacy, and expulsion. Int $\mathbf{J}$ Reprod Contracept Obstet Gynecol. 2012;1(1):26-32.

DOI: $10.18203 / 2320-1770 . i j r \operatorname{cog} 20150118$

Cite this article as: Chauhan M, Guin G. An unusual cause of dyspareunia: secondary cervical perforation by post placental intrauterine contraceptive device. Int J Reprod Contracept Obstet Gynecol 2015;4:881-3. 